\title{
ON A CERTAIN CONVOLUTION OF POLYLOGARITHMS
}

\author{
HIROFUMI TSUMURA
}

(Received 22 November 2011)

\begin{abstract}
In this paper, we consider certain double series analogous to Tornheim's double series and real analytic Eisenstein series. By computing double integrals in two ways, we express the double series as a sum of products of polylogarithms. The technique generalises one given by Kanemitsu, Tanigawa and Yoshimoto. Evaluating the double series at particular points gives new evaluations for certain double series in terms of values of the Riemann zeta function and the dilogarithm which are analogues of formulas of Mordell and Goncharov.
\end{abstract}

2010 Mathematics subject classification: primary 11M41; secondary 11M99.

Keywords and phrases: polylogarithms, Riemann zeta-function, real analytic Eisenstein series.

\section{Introduction}

Let $\mathbb{N}$ be the set of natural numbers, $\mathbb{N}_{0}=\mathbb{N} \cup\{0\}, \mathbb{Z}$ the ring of rational integers, $\mathbb{Q}$ the field of rational numbers, $\mathbb{R}$ the field of real numbers, $\mathbb{R}_{+}$the set of positive real numbers and $\mathbb{C}$ the field of complex numbers. Let $i=\sqrt{-1}$, and $\zeta_{m}=e^{2 \pi i / m}(m \in \mathbb{N})$ be the primitive $m$ th root of unity.

In the 1950s, Tornheim [17] studied the double series defined by

$$
T\left(s_{1}, s_{2}, s_{3}\right)=\sum_{m=1}^{\infty} \sum_{n=1}^{\infty} \frac{1}{m^{s_{1}} n^{s_{2}}(m+n)^{s_{3}}}
$$

for $s_{1}, s_{2}, s_{3} \in \mathbb{N}$, and investigated its interesting properties. Working independently, Mordell [15] showed that $T(2 k, 2 k, 2 k)$ belongs to $\mathbb{Q} \cdot \pi^{6 k}$ for $k \in \mathbb{N}$. Since these works, special values of (1.1) have been actively investigated (for the known results, see [5]).

In [11], Matsumoto considered analytic properties of (1.1) for $\left(s_{1}, s_{2}, s_{3}\right) \in \mathbb{C}^{3}$. Actually he showed that (1.1) can be continued meromorphically to $\mathbb{C}^{3}$ and determined its possible singularities. Based on his result, the author [20] gave some functional relations between (1.1) and the Riemann zeta-function $\zeta(s)$, which contain known

This research was partially supported by Grant-in-Aid for Science Research (No. 23540022), Japan Society for the Promotion of Science.

(c) 2012 Australian Mathematical Publishing Association Inc. 0004-9727/2012 \$16.00 
results given in $[15,17,18]$ and so on. As a more general form, Matsumoto and the author proved the functional relation for double polylogarithms, that is,

$$
\begin{aligned}
\sum_{m, n=1}^{\infty} & \frac{x^{n}}{m^{k} n^{l}(m+n)^{s}}+(-1)^{k} \sum_{m, n=1}^{\infty} \frac{x^{n}}{m^{k} n^{s}(m+n)^{l}}+(-1)^{l} \sum_{m, n=1}^{\infty} \frac{x^{m+n}}{m^{l} n^{s}(m+n)^{k}} \\
= & 2 \sum_{j=0}^{[k / 2]}\left(\begin{array}{c}
k+l-2 j-1 \\
k-2 j
\end{array}\right) \zeta(2 j) \operatorname{Li}(s+k+l-2 j ; x) \\
& +2 \sum_{j=0}^{[l / 2]}\left(\begin{array}{c}
k+l-2 j-1 \\
l-2 j
\end{array}\right) \zeta(2 j) \operatorname{Li}(s+k+l-2 j ; x)
\end{aligned}
$$

for $s \in \mathbb{C}$ with $\operatorname{Re}(s)>0, k, l \in \mathbb{N}$ and $x \in \mathbb{C}$ with $|x| \leq 1$, where

$$
\operatorname{Li}(s ; x)=\sum_{m=1}^{\infty} \frac{x^{m}}{m^{s}}
$$

is the ordinary polylogarithm (see [13, Equation (3.13)]). In particular, when $x=1$, this gives a simple form of the result in [20], which essentially coincides with Nakamura's form (see [16]). Also the above formula gives some of our previous results for double polylogarithms given in [19].

Also, from another viewpoint, it is known that $T(s, s, s)$ can be regarded as the Witten zeta-function associated with the Lie algebra sl(3) (see [22]). Furthermore, (1.1) can be generalised to the multi-variable Witten zeta-function studied by Komori, Matsumoto and the author (see, for example, [7, 8, 12]).

In the present paper, we consider two types of polylogarithmic analogues of (1.1) defined by

$$
\begin{aligned}
& \mathcal{R}\left(s_{1}, s_{2}, s_{3} ; \tau ; x\right)=\sum_{m, n=1}^{\infty} \frac{x^{m+n}(\operatorname{Im}(\tau))^{s_{3}}}{m^{s_{1}} n^{s_{2}}|m-n \tau|^{2 s_{3}}}, \\
& \mathcal{S}\left(s_{1}, s_{2}, s_{3}, s_{4} ; x\right)=\sum_{m, n=1}^{\infty} \frac{x^{m+n}}{m^{s_{1}} n^{s_{2}}\left(m^{s_{3}}+n^{s_{4}}\right)},
\end{aligned}
$$

for $x \in \mathbb{C}$ with $|x| \leq 1$ and $\tau \in \mathbb{C}$ with $\operatorname{Im}(\tau)>0$. Note that (1.2) can also be regarded as an analogue of the real analytic Eisenstein series

$$
E(z, s)=\frac{1}{2} \sum_{\substack{m, n \in \mathbb{Z}^{2} \\(m, n)=1}} \frac{(\operatorname{Im}(\tau))^{s}}{|m-n \tau|^{2 s}}
$$

(see, for example, [9]).

The main aim in this paper is to prove the following two types of functional relations. First, we prove the following theorem. 
Theorem 1.1. Let $q \in \mathbb{N}, l \in \mathbb{N}_{0}, s \in \mathbb{C}$ with $\operatorname{Re}(s)>0, x \in \mathbb{C}$ with $|x| \leq 1$, and $j \in \mathbb{N}_{0}$ with $0 \leq j<q / 2$. Then

$$
\begin{aligned}
\mathcal{R}(s, s & \left.+2 q l+q, 1 ; \zeta_{2 q}^{2 j+1} ; x\right) \\
& =\sum_{m, n=1}^{\infty} \frac{x^{m+n} \operatorname{Im}\left(\zeta_{2 q}^{2 j+1}\right)}{m^{s} n^{s+2 q l+q}\left|m-n \zeta_{2 q}^{2 j+1}\right|^{2}} \\
& =\frac{i}{2} \sum_{v=0}^{(2 l+1) q} \zeta_{2 q}^{-v(2 j+1)} \operatorname{Li}(s+v+1 ; x) \operatorname{Li}(s+2 q l+q+1-v ; x) .
\end{aligned}
$$

For example, in the case $(q, l, j, s, x)=(4,0,0,2,1)$,

$$
\mathcal{R}\left(2,6,1 ; \zeta_{8} ; 1\right)=\sum_{m, n=1}^{\infty} \frac{\operatorname{Im}\left(\zeta_{8}\right)}{m^{2} n^{6}\left|m-n \zeta_{8}\right|^{2}}=\frac{\sqrt{2} \pi^{10}}{170100}+\frac{1}{2} \zeta(5)^{2} .
$$

Next we consider $\mathcal{S}\left(s_{1}, s_{2}, 2 d, 2 d ; x\right)$ for any fixed $d \in \mathbb{N}$, which is convergent absolutely, for example, for $s_{1}, s_{2} \in \mathbb{C}$ with $\operatorname{Re}\left(s_{1}\right)>1-2 d$ and $\operatorname{Re}\left(s_{2}\right)>1$. Then we prove the following theorem.

Theorem 1.2. Let $d \in \mathbb{N}, l \in \mathbb{N}_{0}, s \in \mathbb{C}$ with $\operatorname{Re}(s)>0$ and $x \in \mathbb{C}$ with $|x| \leq 1$. Then

$$
\begin{aligned}
\mathcal{S}(s- & 2 d+1, s+4 d l+1,2 d, 2 d ; x) \\
= & \sum_{m, n=1}^{\infty} \frac{x^{m+n}}{m^{s-2 d+1} n^{s+4 d l+1}\left(m^{2 d}+n^{2 d}\right)} \\
= & \frac{1}{2} \sum_{j=0}^{2 l}(-1)^{j} \operatorname{Li}(s+2 d j+1 ; x) \operatorname{Li}(s+4 d l+1-2 d j ; x) .
\end{aligned}
$$

For example, in the cases $(d, l, s, x)=(1,0,3,1)$ and $(1,0,1,1 / 2)$, we obtain

$$
\begin{gathered}
\mathcal{S}(2,4,2,2 ; 1)=\sum_{m, n=1}^{\infty} \frac{1}{m^{2} n^{4}\left(m^{2}+n^{2}\right)}=\frac{1}{16200} \pi^{8}, \\
\mathcal{S}\left(0,2,2,2 ; \frac{1}{2}\right)=\sum_{m, n=1}^{\infty} \frac{2^{-m-n}}{n^{2}\left(m^{2}+n^{2}\right)}=\frac{1}{2}\left(\frac{\pi^{2}}{12}-\frac{(\log 2)^{2}}{2}\right)^{2}
\end{gathered}
$$

(by (4.6)), which can be regarded as analogues of Mordell's formula as mentioned above, for example,

$$
\sum_{m, n=1}^{\infty} \frac{1}{m^{2} n^{2}(m+n)^{2}}=\frac{1}{2835} \pi^{6}
$$

and of Goncharov's result [4] for double polylogarithms,

$$
\sum_{m, n=1}^{\infty} \frac{2^{-m}}{n(m+n)}=\frac{1}{12} \pi^{2}
$$

(see Remark 4.1). 
We also give a strange formula

$$
\mathcal{S}(-2,4 l, 2,2 ; 1)=-\frac{\pi}{2} \sum_{n=1}^{\infty} \frac{\operatorname{coth}(n \pi)}{n^{4 l-1}} \quad(l \in \mathbb{N}),
$$

where the left-hand side is not convergent (see Proposition 5.1).

It should be noted that (1.2) and (1.3) seem to be associated with the Lie algebra sl(3) like Tornheim's double series (1.1). Therefore it is interesting and meaningful that our present result may be an important step towards showing a certain class of multiple analogues, similarly to the case of Witten zeta-functions as stated above.

\section{Convolution of polylogarithms}

In this section, we consider a certain convolution of polylogarithms. The technique used here is a kind of generalisation of the known one given by Kanemitsu et al. [6].

Let $\xi \in \mathbb{C}$ with $\operatorname{Re}(\xi)>0$ and $|\xi|=1, s \in \mathbb{R}$ with $s>1$ and $x \in \mathbb{C}$ with $|x| \leq 1$. Let

$$
g(u ; s, \xi, x)=\sum_{m=1}^{\infty} \frac{x^{m} e^{-m \xi u}}{(m \xi)^{s}} \quad(u>0)
$$

and

$$
J(k, s, \xi, x)=\int_{0}^{\infty} \int_{0}^{\infty}(u+v)^{k-1} g(u ; s, \xi, x) g(v ; s, \bar{\xi}, x) d u d v \quad(k \in \mathbb{N})
$$

where we denote by $\bar{\xi}$ the complex conjugate of $\xi$, namely $\bar{\xi}=\xi^{-1}$ because $|\xi|=1$. We prove the following two lemmas.

LEMмa 2.1. With the above notation,

$$
J(k, s, \xi, x)=(k-1) ! \sum_{j=0}^{k-1} \xi^{k-1-2 j} \operatorname{Li}(s+j+1 ; x) \operatorname{Li}(s+k-j ; x) .
$$

Proof. Let

$$
\Gamma(z)=\int_{0}^{\infty} u^{z-1} e^{-u} d u \quad(z>0)
$$

be the gamma function. For $\rho \in \mathbb{C}$ with $\operatorname{Re}(\rho)>0$, it is well known that

$$
\Gamma(z)=\int_{0}^{\rho \infty} u^{z-1} e^{-u} d u \quad(z>0)
$$

(see [21, Section 12.20]). Since $\operatorname{Re}(\xi)>0$, we see that for $t>0$,

$$
\begin{aligned}
\int_{0}^{\infty} u^{t-1} g(u ; s, \xi, x) d u & =\sum_{m=1}^{\infty} \frac{x^{m}}{(m \xi)^{s}} \int_{0}^{m \xi \infty}\left(\frac{v}{m \xi}\right)^{t-1} e^{-v} \frac{d v}{m \xi} \\
& =\xi^{-s-t} \Gamma(t) \operatorname{Li}(s+t ; x) .
\end{aligned}
$$


Hence, using the binomial theorem,

$$
\begin{aligned}
J(k, s, \xi, x)= & \int_{0}^{\infty} \int_{0}^{\infty}(u+v)^{k-1} g(u ; s, \xi, x) g(v ; s, \bar{\xi}, x) d u d v \\
= & \sum_{j=0}^{k-1}\left(\begin{array}{c}
k-1 \\
j
\end{array}\right) \int_{0}^{\infty} u^{j} g(u ; s, \xi, x) d u \int_{0}^{\infty} v^{k-1-j} g(v ; s, \bar{\xi}, x) d v \\
= & \sum_{j=0}^{k-1}\left(\begin{array}{c}
k-1 \\
j
\end{array}\right) \xi^{-s-j-1} j ! \operatorname{Li}(s+j+1 ; x) \\
& \times \bar{\xi}^{-s-k+j}(k-1-j) ! \operatorname{Li}(s+k-j ; x) .
\end{aligned}
$$

This completes the proof.

\section{LEMMA 2.2. With the above notation,}

$$
J(k, s, \xi, x)=(k-1) !\left\{\xi^{1-k} \sum_{m, n=1}^{\infty} \frac{x^{m+n}}{m^{s} n^{s+k}\left(m-n \xi^{2}\right)}+\xi^{k-1} \sum_{m, n=1}^{\infty} \frac{x^{m+n}}{m^{s} n^{s+k}\left(m-n \bar{\xi}^{2}\right)}\right\} .
$$

Proof. We see that

$$
\begin{aligned}
J(k, s, \xi, x) & =\int_{0}^{\infty} \int_{0}^{w} w^{k-1} g(w-v ; s, \xi, x) g(v ; s, \bar{\xi}, x) d v d w \\
& =\int_{0}^{\infty} w^{k-1} \sum_{m, n=1}^{\infty} \frac{x^{m+n} e^{-m \xi w}}{(m \xi)^{s}(n \bar{\xi})^{s}} \int_{0}^{w} e^{(m \xi-n \bar{\xi}) v} d v d w \\
& =\int_{0}^{\infty} w^{k-1} \sum_{m, n=1}^{\infty} \frac{x^{m+n}}{(m \xi)^{s}(n \bar{\xi})^{s}}\left\{\frac{e^{-n \bar{\xi} w}}{m \xi-n \bar{\xi}}-\frac{e^{-m \xi w}}{m \xi-n \bar{\xi}}\right\} d w \\
& =\sum_{m, n=1}^{\infty} \frac{x^{m+n} \Gamma(k)}{(m \xi)^{s}(n \bar{\xi})^{s}(m \xi-n \bar{\xi})}\left\{\frac{1}{(n \bar{\xi})^{k}}-\frac{1}{(m \xi)^{k}}\right\} \\
& =(k-1) ! \sum_{m, n=1}^{\infty}\left\{x^{k-1} \frac{x^{m+n}}{m^{s} n^{s+k}\left(m-n \bar{\xi}^{2}\right)}-\xi^{1-k} \frac{x^{m+n}}{m^{s+k} n^{s}\left(m \xi^{2}-n\right)}\right\}
\end{aligned}
$$

Replacing $(m, n)$ with $(n, m)$ in the latter summand on the right-hand side, we obtain (2.1).

\section{Proof of Theorem 1.1}

In this section, from Lemmas 2.1 and 2.2 in the previous section, we give the proof of Theorem 1.1. Combining these lemmas, we can immediately obtain the following proposition. 
Proposition 3.1. Let $\xi \in \mathbb{C}$ with $\operatorname{Re}(\xi)>0$ and $|\xi|=1$, $s \in \mathbb{R}$ with $s>1$ and $x \in \mathbb{C}$ with $|x| \leq 1$. For $k \in \mathbb{N}$,

$$
\begin{gathered}
\xi^{1-k} \sum_{m, n=1}^{\infty} \frac{x^{m+n}}{m^{s} n^{s+k}\left(m-n \xi^{2}\right)}+\xi^{k-1} \sum_{m, n=1}^{\infty} \frac{x^{m+n}}{m^{s} n^{s+k}\left(m-n \bar{\xi}^{2}\right)} \\
=\sum_{v=0}^{k-1} \xi^{k-1-2 v} \operatorname{Li}(s+v+1 ; x) \operatorname{Li}(s+k-v ; x)
\end{gathered}
$$

Using this fact, we prove Theorem 1.1 as follows.

Proof of Theorem 1.1. Let $q \in \mathbb{N}, l \in \mathbb{N}_{0}, s>1, x \in \mathbb{C}$ with $|x| \leq 1$, and $j \in \mathbb{N}_{0}$ with $0 \leq j<q / 2$. Set $\xi=\zeta_{4 q}^{2 j+1}$ and $k=q(2 l+1)+1$ in (3.1). Note that

$$
\xi^{1-k}=\zeta_{4 q}^{-(2 j+1) q(2 l+1)}=\frac{(-1)^{j+l}}{i} .
$$

Hence $\xi^{2 k-2}=-1$. Therefore the left-hand side of (3.1) is equal to

$$
\begin{gathered}
\frac{(-1)^{j+l}}{i} \sum_{m, n=1}^{\infty} \frac{x^{m+n}}{m^{s} n^{s+2 q l+q+1}}\left(\frac{1}{m-n \zeta_{2 q}^{2 j+1}}-\frac{1}{m-n \zeta_{2 q}^{-2 j-1}}\right) \\
=\frac{(-1)^{j+l}}{i} \sum_{m, n=1}^{\infty} \frac{x^{m+n} 2 n i \operatorname{Im}\left(\zeta_{2 q}^{2 j+1}\right)}{m^{s} n^{s+2 q l+q+1}\left|m-n \zeta_{2 q}^{2 j+1}\right|^{2}}
\end{gathered}
$$

On the other hand, the right-hand side of (3.1) is equal to

$$
\begin{aligned}
& \sum_{v=0}^{(2 l+1) q} \zeta_{4 q}^{(2 j+1)(q(2 l+1)-2 v)} \operatorname{Li}(s+v+1 ; x) \operatorname{Li}(s+2 q l+q+1-v ; x) \\
& \quad=(-1)^{j+l} i \sum_{v=0}^{(2 l+1) q} \zeta_{2 q}^{-v(2 j+1)} \operatorname{Li}(s+v+1 ; x) \operatorname{Li}(s+2 q l+q+1-v ; x) .
\end{aligned}
$$

Combining these relations, we see that (1.4) holds for $s>1$. Furthermore, we can easily check that both sides of (1.4) are absolutely convergent for $s \in \mathbb{C}$ with $\operatorname{Re}(s)>0$. Hence the proof of Theorem 1.1 is complete.

\section{Proof of Theorem 1.2}

For $d \in \mathbb{N}$, let $\eta=\zeta_{8 d}=e^{2 \pi i / 8 d}$ be the primitive $8 d$ th root of unity. Since $\eta^{2}=\zeta_{4 d}$ is the primitive $4 d$ th root of unity,

$$
X^{2 d}+Y^{2 d}=\frac{X^{4 d}-Y^{4 d}}{X^{2 d}-Y^{2 d}}=\frac{\prod_{j=0}^{4 d-1}\left(X-\eta^{2 j} Y\right)}{\prod_{j=0}^{2 d-1}\left(X-\eta^{4 j} Y\right)}=\prod_{j=0}^{2 d-1}\left(X-\eta^{2(2 j+1)} Y\right) .
$$


Considering the logarithmic derivative of this equation in $X$,

$$
\begin{aligned}
\frac{2 d X^{2 d-1}}{X^{2 d}+Y^{2 d}} & =\sum_{j=0}^{2 d-1} \frac{1}{X-\eta^{2(2 j+1)} Y} \\
& =\sum_{j=0}^{d-1}\left(\frac{1}{X-\eta^{2(2 j+1)} Y}+\frac{1}{X-\eta^{-2(2 j+1)} Y}\right)
\end{aligned}
$$

by replacing $2 d-1-j(j \geq d)$ with $j$. Using (4.1), we give the proof of Theorem 1.2 as follows.

Proof of Theorem 1.2. We can apply Proposition 3.1 with $\xi=\eta^{2 j+1}=\zeta_{8 d}^{2 j+1}$ for $0 \leq j \leq d-1$ because $\operatorname{Re}(\xi)>0$ for each $\xi$. Setting $k=4 d l+1$ for $l \in \mathbb{N}_{0}$, we have $\xi^{1-k}=\xi^{k-1}=(-1)^{l}$. Therefore it follows from (3.1) with $\xi=\eta^{2 j+1}$ that

$$
\begin{aligned}
& (-1)^{l} \sum_{m, n=1}^{\infty} \frac{x^{m+n}}{m^{s} n^{s+k}}\left(\frac{1}{m-n \eta^{2(2 j+1)}}+\frac{1}{m-n \eta^{-2(2 j+1)}}\right) \\
& \quad=\sum_{v=0}^{4 d l} \eta^{(2 j+1)(4 d l-2 v)} \operatorname{Li}(s+v+1 ; x) \operatorname{Li}(s+4 d l+1-v ; x) \\
& =(-1)^{l} \sum_{v=0}^{4 d l} \eta^{-2 v(2 j+1)} \operatorname{Li}(s+v+1 ; x) \operatorname{Li}(s+4 d l+1-v ; x) .
\end{aligned}
$$

Summing both sides with $j=0,1, \ldots, d-1$ and using (4.1),

$$
\begin{aligned}
& (-1)^{l} \sum_{m, n=1}^{\infty} \frac{x^{m+n}}{m^{s} n^{s+4 d l+1}} \frac{2 d m^{2 d-1}}{m^{2 d}+n^{2 d}} \\
& \quad=(-1)^{l} \sum_{v=0}^{4 d l}\left(\sum_{j=0}^{d-1} \eta^{-2 v(2 j+1)}\right) \operatorname{Li}(s+v+1 ; x) \operatorname{Li}(s+4 d l+1-v ; x) .
\end{aligned}
$$

The left-hand side coincides with $2 d(-1)^{l} \mathcal{S}(s-2 d+1, s+4 d l+1,2 d, 2 d ; x)$. On the other hand, it follows from $\eta^{4 d}=-1$ that

$$
\sum_{j=0}^{d-1} \eta^{-2 v(2 j+1)}= \begin{cases}\frac{2 \eta^{-2 v}}{1-\eta^{-4 v}}=\frac{1}{i \sin (v \pi / 2 d)} & (v \text { is odd }) \\ 0 & 2 d \nmid v, v \text { is even } \\ d(-1)^{v / 2 d} & (2 d \mid v)\end{cases}
$$


Hence the right-hand side of (4.2) can be rewritten as

$$
\begin{aligned}
& (-1)^{l}\left(\sum_{\substack{v=0 \\
v \text { odd }}}^{4 d l} \frac{1}{i \sin (v \pi / 2 d)} \operatorname{Li}(s+v+1 ; x) \operatorname{Li}(s+4 d l+1-v ; x)\right. \\
& \left.\quad+d \sum_{\substack{v=0 \\
2 d \mid v}}^{4 d l}(-1)^{v / 2 d} \operatorname{Li}(s+v+1 ; x) \operatorname{Li}(s+4 d l+1-v ; x)\right)
\end{aligned}
$$

We can confirm that the first sum vanishes by replacing $v$ with $4 d l-v$, because $\sin ((4 d l-v) \pi / 2 d)=-\sin (v \pi / 2 d)$. Hence this is equal to

$$
d(-1)^{l} \sum_{j=0}^{2 l}(-1)^{j} \operatorname{Li}(s+2 d j+1 ; x) \operatorname{Li}(s+4 d l+1-2 d j ; x) .
$$

Therefore (1.5) holds for $s>1$. Furthermore, we can see that both sides of (1.5) are absolutely convergent for $\operatorname{Re}(s)>0$. Thus the proof of Theorem 1.2 is complete.

REMARK 4.1. In [4, Section 1], Goncharov gave a fascinating formula,

$$
\sum_{m, n=1}^{\infty} \frac{x_{1}^{n} x_{2}^{m+n}}{n(m+n)}=\operatorname{Li}\left(2 ; \frac{1-x_{1}}{1-x_{2}^{-1}}\right)-\operatorname{Li}\left(2 ; \frac{1}{1-x_{2}^{-1}}\right)-\operatorname{Li}\left(2 ; x_{1} x_{2}\right) .
$$

In particular, the case $\left(x_{1}, x_{2}\right)=(1 / x, x)$ for $|x|<1$ gives

$$
\sum_{m, n=1}^{\infty} \frac{x^{m}}{n(m+n)}=-\operatorname{Li}\left(2 ; \frac{x}{x-1}\right)
$$

Setting $x=1 / 2$ in (4.3) and using $\operatorname{Li}(2 ;-1)=-\pi^{2} / 12$, we obtain (1.8).

ExAmple 4.2. Setting $(d, l)=(1,0)$ in $(1.5)$,

$$
\mathcal{S}(s-1, s+1,2,2 ; x)=\sum_{m, n=1}^{\infty} \frac{x^{m+n}}{m^{s-1} n^{s+1}\left(m^{2}+n^{2}\right)}=\frac{1}{2} \operatorname{Li}(s+1 ; x)^{2} .
$$

In particular, when $s=1$,

$$
\mathcal{S}(0,2,2,2 ; x)=\sum_{m, n=1}^{\infty} \frac{x^{m+n}}{n^{2}\left(m^{2}+n^{2}\right)}=\frac{1}{2} \operatorname{Li}(2 ; x)^{2},
$$

which can be regarded as an analogue of (4.3). Furthermore, setting $x=1$ and $s=2,3$ in (4.4),

$$
\begin{gathered}
\sum_{m, n=1}^{\infty} \frac{1}{m n^{3}\left(m^{2}+n^{2}\right)}=\frac{1}{2} \zeta(3)^{2}, \\
\sum_{m, n=1}^{\infty} \frac{1}{m^{2} n^{4}\left(m^{2}+n^{2}\right)}=\frac{1}{16200} \pi^{8} .
\end{gathered}
$$


Also, setting $(d, l, s, x)=(1,1,3,1),(1,0,1,-1)$ and $(2,0,5,-1)$ in (1.5),

$$
\begin{gathered}
\sum_{m, n=1}^{\infty} \frac{1}{m^{2} n^{12}\left(m^{2}+n^{2}\right)}=\frac{1451}{229864635000} \pi^{16}, \\
\sum_{m, n=1}^{\infty} \frac{(-1)^{m+n}}{n^{2}\left(m^{2}+n^{2}\right)}=\frac{1}{288} \pi^{4}, \\
\sum_{m, n=1}^{\infty} \frac{(-1)^{m+n}}{m^{2} n^{6}\left(m^{4}+n^{4}\right)}=\frac{961}{1828915200} \pi^{12},
\end{gathered}
$$

by noting that $\operatorname{Li}(s ;-1)=\left(2^{1-s}-1\right) \zeta(s)$. Furthermore, setting $x=i$ in $(1.5)$ and considering its imaginary part,

$$
\sum_{m, n=1}^{\infty} \frac{\chi_{4}(m+n)}{m n^{3}\left(m^{2}+n^{2}\right)}=-\frac{3}{32} \zeta(3) L\left(3, \chi_{4}\right)=-\frac{3 \pi^{3}}{1024} \zeta(3)
$$

where $\chi_{4}$ is the primitive Dirichlet character of conductor 4 and

$$
L\left(s, \chi_{4}\right)=\sum_{n=1}^{\infty} \frac{\chi_{4}(n)}{n^{s}}=\sum_{m=0}^{\infty} \frac{(-1)^{m}}{(2 m+1)^{s}}
$$

is the Dirichlet $L$-function associated with $\chi_{4}$.

ExAMPLE 4.3. As for the dilogarithm and the trilogarithm, we know that, for example,

$$
\begin{gathered}
\operatorname{Li}\left(2, \frac{1}{2}\right)=\frac{\pi^{2}}{12}-\frac{1}{2}(\log 2)^{2}, \\
\operatorname{Li}\left(2, \frac{3-\sqrt{5}}{2}\right)=\frac{\pi^{2}}{15}-\frac{1}{4}\left(\log \left(\frac{3-\sqrt{5}}{2}\right)\right)^{2}, \\
\operatorname{Li}\left(3, \frac{1}{2}\right)=\frac{7}{8} \zeta(3)-\frac{1}{2} \zeta(2) \log 2+\frac{1}{6}(\log 2)^{3}
\end{gathered}
$$

(see [10]). Combining (4.5) and (4.6), we obtain (1.7). Furthermore, setting $(s, x)=$ $(1,(3-\sqrt{5}) / 2)$ and $(2,1 / 2)$ in $(4.4)$, we obtain

$$
\begin{gathered}
\sum_{m, n=1}^{\infty} \frac{((3-\sqrt{5}) / 2)^{m+n}}{n^{2}\left(m^{2}+n^{2}\right)}=\frac{1}{450} \pi^{4}-\frac{(\log ((3-\sqrt{5}) / 2))^{2}}{60} \pi^{2}+\frac{(\log ((3-\sqrt{5}) / 2))^{4}}{32} \\
\sum_{m, n=1}^{\infty} \frac{2^{-m-n}}{m n^{3}\left(m^{2}+n^{2}\right)}=\frac{(\log 2)^{2}}{288} \pi^{4}-\left(\frac{(\log 2)^{4}}{72}+\frac{7 \log 2}{96} \zeta(3)\right) \pi^{2} \\
+\frac{(\log 2)^{6}}{72}+\frac{7(\log 2)^{3}}{48} \zeta(3)+\frac{49}{128} \zeta(3)^{2}
\end{gathered}
$$




\section{Series involving hyperbolic functions}

Setting $x=1$ in (1.5), we see that

$$
\begin{aligned}
\mathcal{S}(s- & 2 d+1, s+4 d l+1,2 d, 2 d ; 1) \\
& =\frac{1}{2} \sum_{j=0}^{2 l}(-1)^{j} \zeta(s+2 d j+1) \zeta(s+4 d l+1-2 d j)
\end{aligned}
$$

holds for $s \in \mathbb{C}$ with $\operatorname{Re}(s)>0, d \in \mathbb{N}$ and $l \in \mathbb{N}_{0}$.

It is well known that $\zeta(s)$ can be continued meromorphically to $\mathbb{C}$ and its singularity is $s=1$ (see [21, Ch. 13]). Hence, by (5.1), $\mathcal{S}(s-2 d+1, s+4 d l+1,2 d, 2 d ; 1)$ can be continued meromorphically to $\mathbb{C}$ and its singularities are $s=-2 d j(0 \leq j \leq 2 l)$. In particular, $\mathcal{S}(s-2 d+1, s+4 d l+1,2 d, 2 d ; 1)$ is holomorphic for $s=-1$, though $s=-1$ does not belong to its convergent region. Setting $s=-1$ and $d=1$ in (5.1),

$$
\mathcal{S}(-2,4 l, 2,2 ; 1)=\frac{(2 \pi)^{4 l}}{8} \sum_{j=0}^{2 l}(-1)^{j} \frac{B_{2 j}}{(2 j) !} \frac{B_{4 l-2 j}}{(4 l-2 j) !},
$$

because

$$
\zeta(2 k)=-\frac{(2 \pi i)^{2 k}}{2(2 k) !} B_{2 k} \quad\left(k \in \mathbb{N}_{0}\right) .
$$

Note that $\left\{B_{n}\right\}$ are the Bernoulli numbers defined by $B_{n}=B_{n}(0)$, where

$$
\frac{t e^{X t}}{e^{t}-1}=\sum_{n=0}^{\infty} B_{n}(X) \frac{t^{n}}{n !}
$$

(see [21, Ch. 13]). On the other hand, it is known that

$$
\sum_{n=1}^{\infty} \frac{\operatorname{coth}(n \pi)}{n^{4 l-1}}=-\frac{(2 \pi)^{4 l-1}}{2} \sum_{j=0}^{2 l}(-1)^{j} \frac{B_{2 j}}{(2 j) !} \frac{B_{4 l-2 j}}{(4 l-2 j) !},
$$

as given by Lerch and recovered by Ramanujan (see Berndt [1, p. 293, Equation (25.3)]). Combining (5.2) and (5.3), we obtain a strange formula as follows. Proposition 5.1. For $l \in \mathbb{N}$,

$$
\mathcal{S}(-2,4 l, 2,2 ; 1)=-\frac{\pi}{2} \sum_{n=1}^{\infty} \frac{\operatorname{coth}(n \pi)}{n^{4 l-1}},
$$

where the left-hand side of (5.4) is defined by analytic continuation using (5.1), while it is not convergent.

REMARK 5.2. Similarly, setting $(d, s, x)=(1,-1,-1)$ in $(1.5)$, and noting that

$$
\operatorname{Li}(2 k ;-1)=\left(2^{1-2 k}-1\right) \zeta(2 k)=-\frac{(2 \pi i)^{2 k}}{2(2 k) !} B_{2 k}\left(\frac{1}{2}\right) \quad\left(k \in \mathbb{N}_{0}\right)
$$


(see [3, Ch. 1]), we obtain

$$
\mathcal{S}(-2,4 l, 2,2 ;-1)=\frac{(2 \pi)^{4 l}}{8} \sum_{j=0}^{2 l}(-1)^{j} \frac{B_{2 j}(1 / 2)}{(2 j) !} \frac{B_{4 l-2 j}(1 / 2)}{(4 l-2 j) !} .
$$

On the other hand, it is known that

$$
\sum_{n=1}^{\infty} \frac{(-1)^{n}}{\sinh (n \pi) n^{4 l-1}}=-\frac{(2 \pi)^{4 l-1}}{2} \sum_{j=0}^{2 l}(-1)^{j} \frac{B_{2 j}(1 / 2)}{(2 j) !} \frac{B_{4 l-2 j}(1 / 2)}{(4 l-2 j) !},
$$

as given by Cauchy [2] and recovered by Mellin [14] and also by Ramanujan (see Berndt [1, p. 294, (25.5)]). Therefore

$$
\mathcal{S}(-2,4 l, 2,2 ;-1)=-\frac{\pi}{2} \sum_{n=1}^{\infty} \frac{(-1)^{n}}{\sinh (n \pi) n^{4 l-1}} \quad(l \in \mathbb{N}) .
$$

It would be interesting to give certain functional relations including (5.4) and (5.5).

\section{Acknowledgement}

The author wishes to express his sincere gratitude to the referee for his/her valuable suggestion.

\section{References}

[1] B. C. Berndt, Ramanujan's Notebooks, Part II (Springer, New York, 1989).

[2] A. Cauchy, Oeuvres Complètes d'Augustin Cauchy, Série II, t. VII (Gauthier-Villars, Paris, 1889).

[3] K. Dilcher, 'Zeros of Bernoulli, generalized Bernoulli and Euler polynomials', Mem. Amer. Math. Soc. 73(386) (1988).

[4] A. B. Goncharov, 'The double logarithm and Manin's complex for modular curves', Math. Res. Lett. 4(5) (1997), 617-636.

[5] J. G. Huard, K. S. Williams and N.-Y. Zhang, 'On Tornheim's double series', Acta Arith. 75(2) (1996), 105-117.

[6] S. Kanemitsu, Y. Tanigawa and M. Yoshimoto, 'Convolution of Riemann zeta-values', J. Math. Soc. Japan 57(4) (2005), 1167-1177.

[7] Y. Komori, K. Matsumoto and H. Tsumura, 'On multiple Bernoulli polynomials and multiple L-functions of root systems', Proc. Lond. Math. Soc. (3) 100(2) (2010), 303-347.

[8] Y. Komori, K. Matsumoto and H. Tsumura, 'On Witten multiple zeta-functions associated with semisimple Lie algebras II', J. Math. Soc. Japan 62(2) (2010), 355-394.

[9] R. P. Langlands, On the Functional Equations Satisfied by Eisenstein Series (Springer, Berlin, 1976).

[10] L. Lewin, Polylogarithms and Associated Functions (North-Holland Publishing Co., Amsterdam, 1981).

[11] K. Matsumoto, 'On the analytic continuation of various multiple zeta-functions', in: Number Theory for the Millennium, II (Urbana, IL, 2000) (A. K. Peters, Natick, MA, 2002), pp. 417-440.

[12] K. Matsumoto and H. Tsumura, 'On Witten multiple zeta-functions associated with semisimple Lie algebras I', Ann. Inst. Fourier (Grenoble) 56(5) (2006), 1457-1504. 
[13] K. Matsumoto and H. Tsumura, 'Functional relations among certain double polylogarithms and their character analogues', Šiauliai Math. Semin. 3(11) (2008), 189-205.

[14] Hj. Mellin, 'Eine Formel für den Logarithmus transcendenter Funktionen von endlichem Geschlecht', Acta Math 25(1) (1902), 165-183.

[15] L. J. Mordell, 'On the evaluation of some multiple series', J. Lond. Math. Soc. 33 (1958), 368-371.

[16] T. Nakamura, 'A functional relation for the Tornheim double zeta function', Acta Arith. 125(3) (2006), 257-263.

[17] L. Tornheim, 'Harmonic double series', Amer. J. Math. 72 (1950), 303-314.

[18] H. Tsumura, 'On some combinatorial relations for Tornheim's double series', Acta Arith. 105(3) (2002), 239-252.

[19] H. Tsumura, 'On certain polylogarithmic double series', Arch. Math. (Basel) 88(1) (2007), 42-51.

[20] H. Tsumura, 'On functional relations between the Mordell-Tornheim double zeta functions and the Riemann zeta function', Math. Proc. Cambridge Philos. Soc. 142(3) (2007), 395-405.

[21] E. T. Whittaker and G. N. Watson, A Course of Modern Analysis, 4th edn (Cambridge University Press, Cambridge, 1996).

[22] D. Zagier, 'Values of zeta functions and their applications', in: First European Congress of Mathematics, Vol. II (Paris, 1992), Progress in Mathematics, 120 (Birkhäuser, Basel, 1994), pp. 497-512.

HIROFUMI TSUMURA, Department of Mathematics and Information Sciences, Tokyo Metropolitan University, 1-1, Minami-Ohsawa,

Hachioji, Tokyo 192-0397, Japan

e-mail: tsumura@tmu.ac.jp 